\title{
ON INTEGRATING FACTORS AND ON CONFORMAL MAPPINGS
}

\author{
BY \\ PHILIP HARTMAN( ${ }^{(1)}$
}

The main results of this paper deal with the existence of (local) "integrating factors" for a linear differential form

$$
\omega=P(x, y) d x+Q(x, y) d y,
$$

that is, for the existence of functions $T \neq 0$ and $w$ such that

$$
\omega=T d w,
$$

in the two cases: (i) $P$ and $Q$ are real-valued and

$$
P^{2}+Q^{2} \neq 0
$$

(ii) $P$ and $Q$ are complex-valued and

$$
\operatorname{Im}(P \bar{Q}) \neq 0 .
$$

In both cases, $x$ and $y$ are real variables, while $T, w$ are real-valued in case (i) but complex-valued in (ii).

In the real case (i), $1 / T$ is what is usually called an integrating factor for $\omega$. The solution of the problem in this case depends on the theory of ordinary differential equations. Part I will deal with this case.

While the problem in the complex case (ii) has the same appearance as the problem in the real case, it is of a very different nature. Its solution depends on an elliptic system of partial differential equations. In fact, the problem is equivalent to the problem of conformalizing the Riemannian metric

$$
d s^{2}=\omega \bar{\omega}=|P|^{2} d x^{2}+2 \operatorname{Re}(P \bar{Q}) d x d y+|Q|^{2} d y^{2} .
$$

The condition (4) implies that (5) is positive definite. (Any positive definite, binary $d s^{2}$ can be factored, in more than one way, into $\omega \bar{\omega}$, where (1) satisfies (4).) The complex case (ii) will be considered in Part II.

In both Parts I and II, it will be supposed that $P$ and $Q$ are continuous. Conditions will then be imposed on the set function

$$
\psi(E)=\int_{J} P d x+Q d y,
$$

Received by the editors October 5, 1956.

1 This research was supported by the United States Air Force through the Air Force Office of Scientific Research of the Air Research and Development Command under contract No. AF 18(603)-41. 
where $E$ is a domain bounded by a rectifiable Jordan curve $J$.

The appendix will consider a related problem on indefinite forms (5) in the theory of surfaces. In particular, this latter problem has applications in the theory of the introduction of asymptotic lines or lines of curvature as coordinate curves.

\section{PART I}

1. The main theorem (III) in this part will be to the effect that if $P, Q$ are real-valued, continuous functions on $x^{2}+y^{2}<1$ satisfying (3) and if there exists a continuous function $f$ satisfying the integral relation

$$
\int_{J} P d x+Q d y=\iint_{E} f d x d y
$$

for every domain $E$ bounded by a rectifiable Jordan curve $J$ in $x^{2}+y^{2}<1$, then (1) has a continuous integrating factor $1 / T(x, y)$ on a sufficiently small circle $x^{2}+y^{2}<\epsilon^{2}$.

It will follow from (II) that the above mentioned conditions on $P, Q$ are sufficient to assure the (local) existence of $C^{1}$-solutions of the Cauchy problem

$$
Q(x, y) z_{x}-P(x, y) z_{y}=0, \quad z(0, y)=z_{0}(y),
$$

if $z_{0}(y)$ is a given $C^{1}$-function. (This reduces to a standard assertion if $Q \equiv 1$ and $f=-P_{y}$ exists and is continuous.)

The proofs of (II) and (III) depend on a new uniqueness theorem (I) for ordinary initial value problems

$$
d y / d x=-P(x, y) / Q(x, y), \quad y(0)=0 .
$$

In particular, (I) implies that the solution of (9) is unique if $P, Q(\neq 0)$ are continuous in a vicinity of the origin and satisfy uniform Lipschitz conditions with respect to $y, x$, respectively. (This is, of course, a standard theorem if $Q \equiv 1$.)

2. A simplified version of the arguments of [6] leads to the following uniqueness theorem $\left({ }^{2}\right)$ for ordinary differential equations:

(I) Let $P(x, y), Q(x, y)$ be real-valued, continuous functions on $x^{2}+y^{2} \leqq 1$ with the properties that

$$
Q(x, y) \neq 0
$$

and that there exists a bounded measurable function $f(x, y)$ in $x^{2}+y^{2} \leqq 1$ such that (7) holds for every rectangle $E$ with boundary $J$ in $x^{2}+y^{2} \leqq 1$. Then the

${ }^{2}$ ) (Added in proof, February 11, 1958). Extensions of (I)-(III) to systems of ordinary differential equations and a converse of (II) in this case are given in the paper On exterior derivatives and solutions of ordinary differential equations to appear in these Transactions. 
initial value problem (9) has a unique solution (for small $|x|$ ).

If the continuous functions $P, Q$ satisfy uniform Lipschitz conditions with respect to $y, x$, respectively, so that $P_{y}, Q_{x}$ exist almost everywhere and are bounded, then (7) holds with $f=Q_{x}-P_{y}$. Hence (I) implies the statement in $\S 1$ concerning (9).

In the still more particular case that $P, Q$ are continuous and that $P_{y}, Q_{x}$ exist and are continuous, the arguments used in [8, pp. 152-153], show that (I) can be deduced from a standard uniqueness theorem by the $C^{1}$ change of variables $(x, y) \rightarrow(x, m)$, where $m(x, y)=\int_{0}^{y} Q(x, t) d t$.

Proof of (I). Suppose that (I) is false and that (9) has two solutions, $y=y_{1}(x)$ and $y=y_{2}(x)$, on $0 \leqq x \leqq \epsilon$ and that $y_{1}(\epsilon)<y_{2}(\epsilon)$. It can be supposed that $y_{1}(x)<y_{2}(x)$ for $0<x \leqq \epsilon$, for otherwise the interval $0 \leqq x \leqq \epsilon$ can be replaced by a sub-interval $x_{0} \leqq x \leqq \epsilon$, where $0 \leqq x_{0}<\epsilon$ and $y_{1}\left(x_{0}\right)=y_{2}\left(x_{0}\right)$.

For a fixed value of $t$, where $0<t \leqq \epsilon$, let $E=E_{\imath}$ denote the $(x, y)$-set $0 \leqq x \leqq t, y_{1}(x) \leqq y \leqq y_{2}(x)$. The assumptions of (I) imply that (7) is valid for $E=E_{t}$, if $J=J_{t}$ is the boundary of $E_{t}$.

Since (9) implies that the line integral of $P d x+Q d y$ is 0 along the arcs $y=y_{1}(x)$ and/or $y=y_{2}(x)$, the relation (7) and Fubini's theorem show that

$$
\int_{y_{1}(t)}^{y_{2}(t)} Q(t, y) d y=\int_{0}^{t}\left(\int_{y_{1}(x)}^{y_{2}(x)} f(x, y) d y\right) d x .
$$

Hence, as $t \rightarrow+0$, the continuity of $Q$ implies that

$$
(Q(0,0)+o(1))\left(y_{2}(t)-y_{1}(t)\right) \leqq \text { Const. } t \max _{0 \leqq x \leqq}\left(y_{2}(x)-y_{1}(x)\right)
$$

if $|f| \leqq$ Const. almost everywhere. Let $t_{1}>t_{2}>\cdots$ be a decreasing sequence of $x$-values such that $t_{n} \rightarrow 0$ as $n \rightarrow \infty$ and that

$$
y_{2}(t)-y_{1}(t)=\max _{0 \leqq x \leqq t}\left(y_{2}(x)-y_{1}(x)\right) \quad \text { if } t=t_{n} .
$$

Since $y_{2}(t)-y_{1}(t)>0$ for $t>0$, the last two formula lines give

$$
Q(0,0)+o(1) \leqq \text { Const. } t \text { if } t=t_{n} \text { and } n \rightarrow \infty \text {. }
$$

This leads to $Q(0,0)=0$, which contradicts (10). This proves (I).

3. A refinement of the assumptions lead to a corresponding refinement of the assertion of (I):

(II) Let $P, Q \neq 0, f$ be real-valued and continuous on $x^{2}+y^{2} \leqq 1$ with the property that (7) holds for all rectangles $E$ in $x^{2}+y^{2} \leqq 1$. Then there exists an $\epsilon>0$ such that the solution $y=y(x, v)$ of

$$
d y / d x=-P(x, y) / Q(x, y) \text { and } y(0, v)=v
$$

exists and is of class $C^{1}$ for $|x| \leqq \epsilon,|v| \leqq \epsilon$.

Proof of (II). It is clear from (I) that there exists an $\epsilon>0$ such that 
$y=y(x, v)$ exists and is continuous for $|x| \leqq \epsilon,|v| \leqq \epsilon$. It also follows from (12) that $y_{x}(x, v)$ exists and is continuous. Thus in order to prove (II), it remains to show that $y_{v}(x, v)$ exists and is continuous.

To this end, let $0<t \leqq \epsilon$ and $-\epsilon \leqq v_{1}<v_{2} \leqq \epsilon$. Let $y_{1}(x)=y\left(x, v_{1}\right)$ and $y_{2}(x)$ $=y\left(x, v_{2}\right), E=E_{t}$ denote the $(x, y)$-set defined by $0 \leqq x \leqq t, y_{1}(x) \leqq y \leqq y_{2}(x)$, and $J=J_{t}$ the boundary of $E$. Since the contribution of the arcs $y=y_{1}(x)$, $y=y_{2}(x)$ to the left side of (7) is 0 by (12), it follows that

$$
\int_{y_{1}(t)}^{y_{2}(t)} Q(t, y) d y-\int_{v_{1}}^{v_{2}} Q(0, y) d y=\int_{0}^{t}\left(\int_{y_{1}(x)}^{y_{2}(x)} f(x, y) d y\right) d x .
$$

The right side of this equation has a continuous derivative with respect to $t$, hence, the left side does also. A differentiation with respect to $t$ gives

$$
\left\{\int_{y_{1}(t)}^{y_{2}(t)} Q(t, y) d y\right\}_{t}=\int_{y_{1}(t)}^{y_{2}(t)} f(t, y) d y,
$$

which, for fixed $v_{1}$ and $v_{2}$, can be written in the form $z^{\prime}(t)=a(t) z(t)$ if

$$
z=\int_{y_{1}(t)}^{y_{2}(t)} Q(t, y) d y \text { and } a=\int_{y_{1}(t)}^{y_{2}(t)} f(t, y) d y / \int_{y_{1}(t)}^{y_{2}(t)} Q(t, y) d y .
$$

Thus $z(t)=z(0) \exp \int_{0}^{t} a(x) d x$. This equation is equivalent to

$$
\int_{y_{1}(t)}^{y_{2}(t)} Q(t, y) d y=\left(\int_{v_{1}}^{v_{2}} Q(0, y) d y\right) \exp \int_{0}^{t} a(x) d x .
$$

It is clear that, as $v_{2} \rightarrow v_{1}$,

$$
\begin{aligned}
& \int_{y_{1}(t)}^{y_{2}(t)} Q(t, y) d y=\left(Q\left(t, y_{1}(t)\right)+o(1)\right)\left(y_{2}(t)-y_{1}(t)\right), \\
& a(t)=a\left(t, v_{2}, v_{1}\right)=f\left(t, y_{1}(t)\right) / Q\left(t, y_{1}(t)\right)+o(1),
\end{aligned}
$$

hold uniformly in $t$ and $v_{1}$. The case $t=0$ of $\left(15_{1}\right)$ is

$$
\int_{v_{1}}^{v_{2}} Q(0, y) d y=\left(Q\left(0, v_{1}\right)+o(1)\right)\left(v_{2}-v_{1}\right)
$$

as $v_{2} \rightarrow v_{1}$. The relations (14) and $\left(15_{1}\right)-\left(15_{3}\right)$ show that $\left(y_{2}(t)-y_{1}(t)\right) /\left(v_{2}-v_{1}\right)$ tends to limit, as $v_{2} \rightarrow v_{1}$, uniformly in $t$ and $v_{1}$.

Since the cases $t \leqq 0$ and/or $v_{2}<v_{1}$ can be treated similarly, it follows that $y_{v}(x, v)$ exists, is continuous and satisfies

$$
Q(x, y(x, v)) y_{v}(x, v)=Q(0, v) \exp \int_{0}^{x} f(t, y(t, v)) / Q(t, y(t, v)) d t .
$$

This proves (II). 
4. It can be mentioned that if the assumptions of (II) are relaxed to those (I) (that is, if $f$ is measurable and bounded, instead of continuous), a considerable portion of (II) remains valid:

(II') Under the conditions of (I), the function $y=y(x, v)$, defined by (12) on some sufficiently small square $|x| \leqq \epsilon,|v| \leqq \epsilon$, has a continuous partial derivative $y_{x}$, satisfies a uniform lower and upper Lipschitz condition with respect to $v$,

$$
0<\text { const. } \leqq\left(y\left(x, v_{2}\right)-y\left(x, v_{1}\right)\right) /\left(v_{2}-v_{1}\right) \leqq \text { Const., }
$$

where $-\epsilon \leqq v_{1}<v_{2}<\epsilon$, and has a partial derivative $y_{v}$ satisfying (16) for $|x| \leqq \epsilon$ for almost all fixed $v$ on $|v| \leqq \epsilon$.

The proof of $\left(\mathrm{II}^{\prime}\right)$ depends on slight modifications of the proof of (II) and will be omitted.

5. An immediate consequence of (II) is the existence of a continuous, nontrivial integrating factor for $P d x+Q d y$.

(III) Under the assumptions (and in the notation) of (II), the transformation $x=u, y=y(u, v)$ is of class $C^{1}$, has a nonvanishing Jacobian on $|u| \leqq \epsilon$, $|v| \leqq \epsilon$ (if $\epsilon>0$ is sufficiently small) and transforms the differential form $\omega=P d x+Q d y$ into

$$
\omega=T d v, \text { where } T=T(u, v) \neq 0
$$

is given by $Q(u, y(u, v)) y_{v}(u, v)$ and

$$
T_{u} \text { exists and is continuous. }
$$

Since $d x=d u$ and $d y=y_{u} d u+y_{v} d v=(-P / Q) d u+y_{v} d v$, the relation (18), where $T=Q(u, y(u, v)) y_{v}(u, v)$, is clear. Finally, (19) follows from (16), where $x=u$.

6. As an application of (III), consider the problem of introducing orthogonal coordinates for a positive definite, binary, Riemannian metric

$$
d s^{2}=E d x^{2}+2 F d x d y+G d y^{2} .
$$

Let the metric be written in the form $d s^{2}=G^{-1}\left\{\left(E G-F^{2}\right) d x^{2}+(F d x+G d y)^{2}\right\}$. Hence, if (III) is applicable to the form $\omega=F d x+G d y$, then there exists a $C^{1}$-mapping $x=u, y=y(u, v)$ of nonvanishing Jacobian transforming (20) into the orthogonal form $d s^{2}=G^{-1}\left\{\left(E G-F^{2}\right) d u^{2}+T^{2} d v^{2}\right\}$.

The statement (III) can also be applied in the problem of reducing an indefinite quadratic differential form $L d x^{2}+2 M d x d y+N d y^{2}$ to the normal form $2 M^{\prime} d u d v$. To this end, write the given form as

$$
(M+\mu)^{-1}(L d x+M d y+\mu d y)(\mu d x+M d x+N d y) \text {, where } \mu=\left(M^{2}-L N\right)^{1 / 2},
$$

and apply (III), if possible, to the linear differential forms in this expression.

For example, if the quadratic form involved is the second fundamental form of a surface $S$ of class $C^{2}$ and $(x, y)$ are Gaussian parameters in a $C^{2}$ parametrization of $S$, the problem just mentioned is that of introducing 
asymptotic lines as parameter lines on a surface $S$ of negative Gaussian curvature $K$. In this case, there exist continuous functions $f$ satisfying (7) if $P d x+Q d y$ is either of the forms $L d x+M d y$ or $M d x+N d y$; this fact is an integrated form of the Codazzi equations; [7, pp. 759-760]. Thus (III) is applicable to each of the factors $L d x+M d y+\mu d y, \mu d x+M d x+N d y$ if $\mu^{2}$ $=M^{2}-L N$, that is, if the Gaussian curvature $K$ of $S$, has continuous partial derivatives. This implies the result of $[8$, p. 152].

\section{PART II}

7. The main theorem (IV) of this part will imply that if $P, Q$ are complexvalued, continuous functions on $x^{2}+y^{2} \leqq 1$ satisfying (4) and if there exists a function $f$ of class $L^{p}\left(x^{2}+y^{2}<1\right), p>2$, satisfying the integral relation (7) for every domain $E$ bounded by a rectifiable Jordan curve $J$ in $x^{2}+y^{2} \leqq 1$, then there exists a $C^{1}$-mapping

$$
x=x(u, v), \quad y=y(u, v)
$$

on some circle $u^{2}+v^{2}<\epsilon^{2}$, satisfying

$$
x(0,0)=y(0,0)=0,
$$

having a nonvanishing Jacobian, and transforming (1) into the form (2), where $w=u+i v$. (The inequality $T \neq 0$ is implied by $(4)$ and $\partial(x, y) / \partial(u, v) \neq 0$.) In particular, the continuity of $P, Q$ and the existence and boundedness of $Q_{x}, P_{y}$ is sufficient for the existence of such a mapping. This type of condition is very different from the standard type of condition involving the degree of continuity of $P$ and $Q$.

The proof of the sufficiency of the conditions mentioned (and, in fact, of somewhat more general conditions) will depend on the Hölder continuity of quasi-conformal mappings and on some simple facts in potential theory.

An analogue of the italicized assertions, at a higher level of differentiability, is the result of [4] to the effect that if a positive definite binary Riemannian $d s^{2}$ is of class $C^{1}$ and has a continuous curvature, then it can be conformalized by a mapping of class $C^{2}$.

8. The relations (1) and (2) are equivalent to $P x_{u}+Q y_{u}=-i\left(P x_{v}+Q y_{v}\right)$. If (4) holds, this condition is equivalent to the real system of quasi-linear elliptic partial differential equations

$$
x_{u}=B x_{v}+C y_{v}, \quad y_{u}=-A x_{v}-B y_{v},
$$

where $A, B, C$ are functions of $x, y$ and are given by

$$
A=|P|^{2} / \operatorname{Im}(P \bar{Q}), \quad B=\operatorname{Re}(P \bar{Q}) / \operatorname{Im}(P \bar{Q}), \quad C=|Q|^{2} / \operatorname{Im}(P \bar{Q}) .
$$

In particular, $A C-B^{2} \equiv 1$. The mapping

$$
u=u(x, y), \quad v=v(x, y),
$$

inverse to (21), satisfies the linear system 


$$
v_{x}=B u_{x}-A u_{y}, \quad v_{y}=C u_{x}-B u_{y} .
$$

If $P, Q$ are continuous and (4) holds on $x^{2}+y^{2} \leqq 1$, then there exists a constant $k, 0<k<1$, with the property that, for $x^{2}+y^{2} \leqq 1$,

$$
A \xi^{2}+2 B \xi \eta+C \eta^{2} \geqq\left(2 k /\left(1+k^{2}\right)\right)\left(\xi^{2}+\eta^{2}\right) ;
$$

so that (23) implies

$$
x_{u}^{2}+x_{v}^{2}+y_{u}^{2}+y_{v}^{2} \leqq\left(\left(1+k^{2}\right) / k\right)\left(x_{u} y_{v}-x_{v} y_{u}\right) .
$$

The inequality (28) implies that the maximal dilatation $[1$, p. 1$]$ of the mapping (21) does not exceed $1 / k$, that is, that an infinitesimal $(u, v)$-circle is mapped onto an infinitesimal $(x, y)$-ellipse and the ratio of the axes of this ellipse does not exceed $1 / k$; cf. [12, pp. 135-136].

The notation $w=u+i v$ and $z=x+i y$ will be used below; for example, (25) will sometimes be abbreviated as $w=w(z)$, even though $w$ is not an analytic function of $z$.

LemMA 1. Let $0<k \leqq 1$ and let (21) be a one-to-one mapping of $|w|<\epsilon$ onto $|z|<\epsilon$ with the property that (22) holds and that $x, y$ are of class $C^{1}$ and satisfy (28) for $|w|<\epsilon$. Then $z=z(w)$ satisfies a uniform Hölder condition of order $k$, that is, if $w_{1}, w_{2}$ correspond to $z_{1}, z_{2}$, then

$$
\left|z_{1}-z_{2}\right| \leqq M\left|w_{1}-w_{2}\right|^{k} .
$$

Furthermore, the constant $M$ depends only on $k$ and $\epsilon$ (and is otherwise independent of the mapping).

Since the first assertion of Lemma 1 implies that $x, y$ are uniformly continuous on $|w|<\epsilon$, these functions can be supposed to be defined and continuous on $|w| \leqq \epsilon$.

Lemma 1 is contained in the proof of Theorem 5 in Ahlfors [1, pp. 11-13]; cf. Lavrentieff $[11, \S 1]$, where an analogue of Lemma 1 is also given. An earlier similar result of Morrey [12, pp. 136-137], can be used below in place of Lemma 1. A related theorem of Nirenberg [14, pp. 117-122], dealing with an inequality more general than (28), does not seem so convenient, since the order of the Hölder condition is only claimed to be $k /\left(1+k^{2}\right)$.

Since condition (28) of Lemma 1 can be replaced [1] by the assumption that the maximal dilatation does not exceed $1 / k$, Lemma 1 implies that the mapping (25) inverse to (21) satisfies

$$
\left|w_{1}-w_{2}\right| \leqq M\left|z_{1}-z_{2}\right|^{k} .
$$

9. The following result in potential theory will be used in the proof of (IV), below.

Lемма 2. Let $\alpha=\alpha(r)$ be a nondecreasing positive function for $r>0$ satisfying $(\alpha(+0)=0$ and $)$ 


$$
\int_{+0} r^{-1} \alpha(r) d r<\infty
$$

Let $\phi(E)$ be a completely additive set function on the $(u, v)$-plane with support on $u^{2}+v^{2} \leqq a^{2}$ having the property that

$$
\int_{E}|d \phi| \leqq C r \alpha(r) \quad(C=\text { Const. })
$$

if $E$ is contained in a circle of radius $r$. Let $V=V(u, v)$ denote the logarithmic potential

$$
V(u, v)=\iint_{|\zeta|<a} \log |w-\zeta| d_{\zeta} \phi(E),
$$

where $w=u+i v$ and $\zeta=\xi+i \eta$. Then $V(u, v)$ is of class $C^{1}$ on $u^{2}+v^{2}<a^{2}$ and there exists a constant $c$ such that

$$
\begin{gathered}
|V|,\left|V_{u}\right|,\left|V_{v}\right| \leqq c \\
\left|V_{u}\left(w_{1}\right)-V_{u}\left(w_{2}\right)\right|,\left|V_{v}\left(w_{1}\right)-V_{v}\left(w_{2}\right)\right| \leqq c \rho\left(\left|w_{1}-w_{2}\right|\right),
\end{gathered}
$$

where

$$
\rho(r)=\alpha(4 r)+\int_{0}^{4 r} t^{-1} \alpha(t) d t+r \int_{r / 2}^{2 a} t^{-2} \alpha(t) d l .
$$

It is clear that the assumptions on $\alpha(r)$ imply that

$$
0<\text { Const. } r<\rho(r) \rightarrow 0 \text { as } r \rightarrow 0,
$$

in fact, that each term on the right of (36) tends to 0 with $r$ and that the last term is not less than Const. $r$ for small $r$.

This lemma is an analogue of a result of Morrey [13, pp. 52-53], in which $r \alpha(r)$ is replaced by $\alpha(r)$ in the assumption (32) and in which the assertion is correspondingly weaker than in Lemma 2.

Proof of Lemma 2. It will be shown that there exists $a$ constant $c$ such that

$$
|I| \leqq c
$$

and that

$$
\left|I\left(w_{1}\right)-I\left(w_{2}\right)\right| \leqq c \rho\left(\left|w_{1}-w_{2}\right|\right),
$$

if $I=I(w)$ denotes either of the two integrals

(40) $\iint_{|\zeta|<a}(u-\xi)|w-\zeta|^{-2} d_{\zeta} \phi(E), \quad \iint_{|\zeta|<a}(v-\eta)|w-\zeta|^{-2} d_{\zeta} \phi(E)$.

The proof of this fact depends on standard techniques in potential theory. 
It will be clear from the proof that these standard arguments can be used to show that $V$ is of class $C^{1}$ and that the integrals in (40) are $V_{u}, V_{v}$, respectively; so that Lemma 2 follows.

Let $m(r)=m(r ; w)$ denote the integral

$$
m(r)=m(r ; w)=\iint_{|w-\zeta| \leqq r}\left|d_{\zeta} \phi(E)\right| .
$$

Then, if $I(w)$ denotes either of the integrals in (40),

$$
|I(w)| \leqq \int_{0}^{2 a} r^{-1} d m(r) \leqq m(2 a) / 2 a+\int_{0}^{2 a} r^{-2} m(r) d r .
$$

On the other hand, the condition on (32) and the definition (41) of $m(r)$ show that $m(r) \leqq C r \alpha(r)$. Hence,

$$
|I(w)| \leqq C\left\{\alpha(2 a)+\int_{0}^{2 a} r^{-1} \alpha(r) d r\right\} .
$$

This proves the assertion concerning (38).

In order to prove (39), let

$$
h=2\left|w_{1}-w_{2}\right| .
$$

Let $F$ denote the $\zeta$-set defined by

$$
F:\left|\zeta-w_{1}\right|<h, \quad|\zeta|<a
$$

and $G$, the complement of $F$ with respect to $|\zeta|<a$. For any function $g=g(w)$, let $\Delta g$ denote the difference $g\left(w_{2}\right)-g\left(w_{1}\right)$. Then $\Delta I=\Delta I_{1}+\Delta I_{2}$ if

$$
I_{1}=\iint_{F} \cdots \text { and } I_{2}=\iint_{G} \cdots,
$$

the integrands being the same as in the definition of $I$.

The derivation of (42) shows that

$$
\left|I_{1}\left(w_{1}\right)\right| \leqq C\left\{\alpha(h)+\int_{0}^{h} r^{-1} \alpha(r) d r\right\}
$$

and that

$$
\left|I_{1}\left(w_{2}\right)\right| \leqq C\left\{\alpha(2 h)+\int_{0}^{2 h} r^{-1} \alpha(r) d r\right\},
$$

since $\left|\zeta-w_{2}\right| \leqq 2 h$ if $\zeta$ is in $F$. Thus

$$
\left|\Delta I_{1}\right| \leqq 2 C\left\{\alpha(2 h)+\int_{0}^{2 h} r^{-1} \alpha(r) d r\right\} .
$$


By the mean value theorem of differential calculus,

$$
|\Delta(\cdots)| \leqq \text { const. }\left|w_{1}-w_{2}\right| /\left|w_{*}-\zeta\right|^{2},
$$

where $(\cdots)$ denotes the integrand of either integral in (40) and $w_{*}$ $=w_{*}\left(w_{1}, w_{2}, \zeta\right)$ is a point on the line segment joining $w_{1}$ and $w_{2}$. If $\zeta$ is not in $F$, so that $\left|w_{1}-\zeta\right| \geqq 2\left|w_{1}-w_{2}\right|$, then $\left|w_{1}-w_{*}\right| \leqq\left|w_{1}-w_{2}\right| \leqq\left|w_{1}-\zeta\right| / 2$. But $\left|w_{*}-\zeta\right| \geqq\left|w_{1}-\zeta\right|-\left|w_{*}-w_{1}\right|$ and, hence, $\left|w_{*}-\zeta\right| \geqq\left|w_{1}-\zeta\right| / 2$. Consequently, the expression on the right side of the last formula line does not exceed const. $(h / 2)\left|w_{1}-\zeta\right|^{-2}$. The arguments leading to (42) show that

$$
\left|\Delta I_{2}\right| \leqq 2 C \text { const. } h\left\{\alpha(2 a) / 2 a+\int_{h}^{2 a} r^{-2} \alpha(r) d r\right\} \text {. }
$$

The inequalities (45), (46) and the relation $I=I_{1}+I_{2}$ imply the statement concerning (39).

In view of the remarks at the beginning of the proof, this completes the proof of Lemma 2.

10. Lemmas 1 and 2 will now be used to obtain a priori estimates for degrees of continuity belonging to the partial derivatives of solutions of the system (23).

Lemma 3. Let $k, \lambda$ denote real numbers satisfying

$$
0<k<1, \quad 0<\lambda<1, \quad k(1+\lambda)>1 .
$$

Let $P(x, y), Q(x, y)$ be complex-valued, continuous functions on $x^{2}+y^{2} \leqq \epsilon^{2}$ satisfying (27), where $A, B, C$ are given by (24). Let there exist a completely additive set function $\psi(E)$ on the $(x, y)$-plane with support on $x^{2}+y^{2} \leqq \epsilon^{2}$ satisfying

$$
\iint_{E}|d \psi(E)| \leqq \text { Const. } r^{1+\lambda} \quad(0<\lambda<1),
$$

if the set $E$ is contained in a circle of radius $r$, and the integral relation

$$
\int_{J} P d x+Q d y=\psi(E)
$$

if $E$ is a domain bounded by a rectifiable Jordan curve $J$ in $x^{2}+y^{2} \leqq \epsilon^{2}$. Finally, let (21) be a one-to-one mapping of $|w|<\epsilon$ onto $|z|<\epsilon$ of class $C^{1}$ satisfying (22) and transforming (1) into the normal form (2). Then, for every $\delta$, where $0<\delta<\epsilon$, there exists a constant $M$, depending on $k, \lambda, \delta$, the Const. in (48) and upper bounds for $|P|,|Q|$ and $|\operatorname{Im}(P \bar{Q})|^{-1}$ (but otherwise independent of $P, Q$ and the solution (21)), such that the partial derivatives of (21) satisfy

$$
\left|z_{u}(w)\right|,\left|z_{v}(w)\right| \leqq M
$$

and 


$$
\partial(x, y) / \partial(u, v) \geqq 1 / M
$$

if $|w| \leqq \delta$ and

$$
\begin{aligned}
& \left|z_{u}\left(w_{1}\right)-z_{u}\left(w_{2}\right)\right|,\left|z_{v}\left(w_{1}\right)-z_{v}\left(w_{2}\right)\right| \\
& \quad \leqq M\left(\left|w_{1}-w_{2}\right|^{\lambda}+\rho_{0}\left(M\left|w_{1}-w_{2}\right|\right)\right)
\end{aligned}
$$

if $\max \left(\left|w_{1}\right|,\left|w_{2}\right|\right) \leqq \delta$ and $\rho_{0}(r)$ is a monotone majorant for the degrees of continuity of $P$ and $Q$.

The condition on $\rho_{0}$ means that $\rho_{0}(r)$ is a nondecreasing, non-negative function of $r$ satisfying

$$
\left|P\left(z_{1}\right)-P\left(z_{2}\right)\right|,\left|Q\left(z_{1}\right)-Q\left(z_{2}\right)\right| \leqq \rho_{0}\left(\left|z_{1}-z_{2}\right|\right) .
$$

In the proof of Lemma 3 , it is clear that there is no loss of generality in supposing that $P, Q$ are smooth (say, of class $C^{1}$ ). For otherwise, $P, Q, \psi$ can be approximated by the respective functions $P_{n}, Q_{n}, \psi_{n}$ obtained by convoluting $P, Q, \psi$ with $n^{2} K(x / n, y / n)$, where $K=K(x, y)$ is a smooth function for all $(x, y), K \geqq 0, K \equiv 0$ if $x^{2}+y^{2} \geqq 1$ and $\iint K d x d y=1$. On the one hand, the conditions imposed on $P, Q, \psi$ hold for $P_{n}, Q_{n}, \psi_{n}$ with the same $k, \lambda$, Const., $\rho_{0}(r)$ and upper bounds for $\left|P_{n}\right|,\left|Q_{n}\right| ;$ cf. the argument in [2, pp. 62-63]. On the other hand, the assertions will not be altered by a limit process; cf. the proof of (IV) below.

In the case that $P$ and $Q$ are smooth, $\psi(E)$ is the absolutely continuous function

$$
\psi(E)=\iint_{E}\left(Q_{x}-P_{y}\right) d x d y .
$$

Proof of Lemma 3. Let $T=T(u, v)$ denote the function satisfying

$$
P d x+Q d y=T d w,
$$

by virtue of (21); so that,

$$
T=P x_{u}+Q y_{u}=-i\left(P x_{v}+Q y_{v}\right) .
$$

Let $\phi(E)$ be the absolutely additive set function in the $(u, v)$-plane which is the image of $\psi$, that is, $\phi(E)=\psi\left(E^{\prime}\right)$ if $E$ is the $(u, v)$-image of the $(x, y)$-set $E^{\prime}$. Then (49) and (53) show that

$$
\int_{J} T d w=\phi(E)
$$

if $E$ is a domain with a rectifiable Jordan boundary $J$ in $u^{2}+v^{2}<\epsilon^{2}$.

It follows that if $|w|<a<\epsilon$, then

$$
2 \pi i T(w)=\int_{|\zeta|=a}(\zeta-w)^{-1} T(\zeta) d \zeta-\iint_{|\zeta|<a}(\zeta-w)^{-1} d_{\zeta} \phi(E) .
$$


(The relation (57) is a standard Green formula when $\psi$, hence $\phi$, is absolutely continuous with a continuous density; cf., e.g., $[9$, p. 555].) Let the relation (57) be integrated with respect to $a$ over the interval $[a, b]$ and let the result be divided by $b-a$ to give

$$
2 \pi i T(w)=\Phi(w)+\Psi(w),
$$

where

$$
\Phi(w)=\Phi_{a b}(w)=(b-a)^{-1} \int_{a}^{b} d r \int_{|\zeta|=r}(\zeta-w)^{-1} T(\zeta) d \zeta
$$

and

$$
\Psi(w)=\Psi_{a b}(w)=(b-a)^{-1} \int_{a}^{b} \int_{|\zeta|<r}(\zeta-w)^{-1} d_{\zeta} \phi(E) .
$$

The function (59), which is regular analytic for $|w|<a$, is given by

$$
\Phi(w)=i(b-a)^{-1} \int_{a}^{b} \int_{0}^{2 \pi}\left(r e^{i \theta}-w\right)^{-1} T\left(r e^{i \theta}\right) d \theta d r .
$$

Hence, if $|w| \leqq \delta<a$,

$$
|\Phi(w)| \leqq(a-\delta)^{-1} a^{-1}(b-a)^{-1} \iint_{a<|\zeta|<b}|T(\zeta)| d \xi d \eta .
$$

In view of Schwarz's inequality,

$$
(b-a)^{-1} \iint_{a<|\zeta|<b}|T(\zeta)| d \xi d \eta \leqq\left\{2 \pi(b-a)^{-1} \iint_{|\zeta|<\epsilon}|T(\zeta)|^{2} d \xi d \eta\right\}^{1 / 2} \cdot
$$

By the relation (55),

$$
|T|^{2} \leqq \text { const. }\left(x_{u}^{2}+y_{u}^{2}+x_{v}^{2}+y_{v}^{2}\right)
$$

if $|P|,|Q| \leqq$ const. Hence, by (28),

$$
|T|^{2} \leqq \text { const. }(2 / k)\left(x_{u} y_{2}-x_{v} y_{u}\right) \text {. }
$$

Consequently,

$$
\iint_{|\zeta|<\epsilon}|T|^{2} d \xi d \eta \leqq \text { const. }(2 / k) \iint_{|w|<\epsilon} \partial(x, y) / \partial(u, v) d u d v,
$$

where the last integral is merely the integral of $d x d y$ over the disk $|z|<\epsilon$. This gives

$$
\left.|\Phi(w)| \leqq 2 \pi \epsilon(a-\delta)^{-1} a^{-1} \text { const. } /(b-a) k\right)^{1 / 2} \text { if }|w| \leqq \delta<a
$$

and $a<b<\epsilon$. Similarly, 


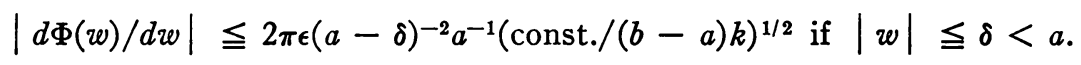

Since Lemma 1 implies that $z=x+i y$ satisfies (29), it follows from (48) that

$$
\iint_{E}|d \phi(E)| \leqq \text { Const. }\left(M r^{k}\right)^{1+\lambda}
$$

if the set $E$ is contained in a circle $\left|z-z_{0}\right| \leqq r$ of radius $r$.

Denote by $\alpha(r)$ the function

$$
\alpha(r)=r^{k(1+\lambda)-1} .
$$

In view of (47), this function satisfies the conditions of Lemma 2. Thus, Lemma 2 is applicable to the logarithmic potential $V(w)=V(w, a)$, given by (33), for any value of $a$ not exceeding $b$. On the other hand, the function (60) is

$$
\Psi(w)=(b-a)^{-1} \int_{a}^{b}\left(V_{u}(w, r)-i V_{v}(w, r)\right) d r .
$$

Since the estimates (34) and (35) apply uniformly to $V(w, r)$ for $|w| \leqq r$ and $a \leqq r \leqq b$, it follows that

$$
|\Psi(w)| \leqq c \text { if }|w| \leqq a
$$

and that

$$
\left|\Psi\left(w_{1}\right)-\Psi\left(w_{2}\right)\right| \leqq c \rho\left(\left|w_{1}-w_{2}\right|\right) \text { if } \max \left(\left|w_{1}\right|,\left|w_{2}\right|\right) \leqq r,
$$

where the function $\rho$ is given by (36) and (65) and the constant $c$ depends only on $\lambda, k, b$, and the Const. in (48).

It follows from (58), (62) and (66) that

$$
|T(w)| \leqq M \text { if }|w| \leqq \delta \quad(<a<b<\epsilon),
$$

where $M$ is a constant depending only on $k, \lambda, \delta, a, b$, upper bounds for $|P|$, $|Q|$, and on the Const. in (48). In view of (55), this proves the assertion concerning (50) in Lemma 3.

The assertion (50) implies that the estimate (29) can be replaced by

$$
\left|z_{1}-z_{2}\right| \leqq M\left|w_{1}-w_{2}\right|
$$

if $M=M(\delta)$ and $\max \left(\left|w_{1}\right|,\left|w_{2}\right|\right) \leqq \delta<\epsilon$. This means that (64) can be improved to

$$
\iint_{E}|d \phi(E)| \leqq \text { Const. }(M r)^{1+\lambda} .
$$

Thus (65) can be replaced by

$$
\alpha(r)=r^{\lambda}
$$


and the function $\rho(r)$ in (36) and (67) is merely an absolute constant times $r^{\lambda}$. Consequently, (63) and (67) imply that

$$
\left|T\left(w_{1}\right)-T\left(w_{2}\right)\right| \leqq M\left|w_{1}-w_{2}\right|^{\lambda} .
$$

Finally, the assertion concerning (52) in Lemma 3 follows from (50), (69) and (71), if it is noted that $P$ and $Q$, as functions of $w$, have a degree of continuity majorized by $\rho_{0}(M r)$; cf. (69).

In order to prove (51), let $0<\delta<\epsilon$ and let $|T(w)|$ assume its minimum value $M_{0}$ on $|w| \leqq \delta$ at the point $w=w_{0}$. Then (71) implies that

$$
|T(w)| \leqq M_{0}+M\left|w-w_{0}\right|^{\lambda} \text { if }|w| \leqq \delta
$$

and the number $M$ depends on the quantities specified in the statement of Lemma 3. The relations (55) and (72) imply that $\left|z_{u}\right|,\left|z_{v}\right|$ do not exceed const. $\left(M_{0}+M \eta^{\lambda}\right)$ if $\left|w-w_{0}\right| \leqq \eta,|w| \leqq \delta$ and const. depends only on upper bounds_for $|P|,|Q|$ and $|\operatorname{Im}(P \bar{Q})|^{-1}$. Hence

$$
\left|z-z_{0}\right| \leqq \text { const. }\left(M_{0}+M \eta^{\lambda}\right)\left|w-w_{0}\right|
$$

if $z=z(w), z_{0}=z\left(w_{0}\right)$ and $\left|w-w_{0}\right| \leqq \eta,|w| \leqq \delta$.

In view of the inequality (30), it follows that

$$
M^{-1 / k}\left|w-w_{0}\right|^{1 / k} \leqq \text { const. }\left(M_{0}+M \eta^{\lambda}\right)\left|w-w_{0}\right| .
$$

Notice that $M$ in (30) depends only on $\epsilon, k, \delta$ since $|w| \leqq \delta$ implies, by (24), that $z(w)$ is contained in a circle $|z| \leqq r$ with a radius $r$ depending on $\epsilon, k, \delta$. The $M$ in the last relation is the maximum of the one occurring in (30) and in (72).

Choose $w$ so that $\left|w-w_{0}\right|=\eta$ and $|w| \leqq \delta$. Then the last inequality gives

$$
M_{0} \geqq(\text { const. })^{-1} M^{-1 / k} \eta^{(1-k) / k}-M \eta^{\lambda} \text {. }
$$

In view of $(47), \lambda>(1-k) / k$. Hence, there exists a small $\eta=\eta(\delta)>0$ with the property that the expression on the right side of the last inequality is positive. Since $|T(w)| \geqq M_{0}$ for $|w| \leqq \delta$, the assertion concerning (51) follows from (61). This completes the proof of Lemma 3.

11. Lemma 3 leads at once to an existence theorem for the "conformal" normal form $T d w$ for $P d x+Q d y$ (or, equivalently, to an existence theorem for the linear elliptic system (24), (26)).

(IV) Let $P, Q$ be complex-valued, continuous functions on $x^{2}+y^{2} \leqq 1$ satisfying $\operatorname{Im}(P \bar{Q}) \neq 0$. Let there exist a completely additive set function $\psi(E)$ on $x^{2}+y^{2} \leqq 1$ satisfying (48) if the set $E$ is contained in a circle of radius $r$ and satisfying the integral relation (49) if $E$ is a domain bounded by a rectifiable Jordan curve $J$ in $x^{2}+y^{2} \leqq 1$. Then, for sufficiently small $\epsilon>0$, there exist oneto-one $C^{1}$-mappings (21) of $u^{2}+v^{2}<\epsilon^{2}$ onto $x^{2}+y^{2}<\epsilon^{2}$ transforming $P d x+Q d y$ into the normal form $T(d u+i d v)$, where

$$
T=T(u, v) \neq 0 .
$$


It can also be assumed that $z(0,0)=0$, in which case, estimates for the degrees of continuity of the partial derivatives of $x, y$ and of $u, v$ are supplied by Lemma 3 .

If $f=f(x, y)$ is of class $L^{p}$ on $x^{2}+y^{2} \leqq 1$ and $p>1$, then

$$
\iint_{E}|f| d x d y \leqq\left(\iint_{E}|f|^{p} d x d y\right)^{1 / p}\left(\pi r^{2}\right)^{1 / q} \leqq \text { Const. } r^{2 / q}
$$

provided that $E$ is contained in a circle of radius $r$ and $1 / p+1 / q=1$. It follows that the conditions (48) and (49) on $\psi$ are satisfied if there exists an $f$ of class $L^{p}, p>2$, satisfying (7); in this case,

$$
\psi(E)=\iint_{E} f d x d y
$$

and $1+\lambda=2 / q>1$.

Proof of (IV). If $x, y$ are first subjected to an affine transformation, it can be supposed that $P(0,0)=1, Q(0,0)=i$. Hence, if $k$ is an arbitrary number on the range $0<k<1$, it follows from (24) and continuity considerations that (27) holds for $x^{2}+y^{2} \leqq \epsilon^{2}$, provided that $\epsilon=\epsilon(k)>0$ is sufficiently small.

If $\lambda$ satisfies (48), choose the number $k$ so as to satisfy (47). Let $\epsilon>0$ be fixed by the requirement that (25) holds for $x^{2}+y^{2} \leqq \epsilon^{2}$.

Let $P, Q, \psi$ be approximated by functions $P_{n}, Q_{n}, \psi_{n}$ described after the statement of Lemma 3. If $P=P_{n}, Q=Q_{n}$, then there exist mappings $x$ $=x_{n}(u, v), y=y_{n}(u, v)$ of the type desired. It can also be supposed that $x_{n}(0,0)=y_{n}(0,0)=0$. Then Lemma 3 implies the uniform boundedness and equicontinuity of $z=z_{n}(u, v)$ and its first order partial derivatives on every circle $u^{2}+v^{2} \leqq \delta^{2}<\epsilon^{2}$. Thus, it is possible to extract a subsequence of $z_{1}, z_{2}, \ldots$ which, together with the first order partial derivatives, have uniform limits on every circle $u^{2}+v^{2} \leqq \delta^{2}<\epsilon^{2}$.

The limit function $z=z(u, v)$ is of class $C^{1}$ on $u^{2}+v^{2}<\epsilon^{2}$. It is easy to see, from the estimates (29) and (30) for the mapping $z=z_{n}(u, v)$ and its inverse, that $z=z(u, v)$ is a one-to-one mapping (29) of $u^{2}+v^{2}<\epsilon^{2}$ onto $x^{2}+y^{2}<\epsilon^{2}$; $[1$, p. 14$]$.

Finally, the uniformity of the estimate (51), with respect to $n$, shows that $\partial(x, y) / \partial(u, v)>0$ on $u^{2}+v^{2}<\epsilon^{2}$. This implies (73) and completes the proof of (IV).

12. As pointed out at the beginning of the paper, (IV) states that a positive definite, binary Riemannian metric

$$
d s^{2}=E d x^{2}+2 F d x d y+G d y^{2}
$$

can be reduced to the conformal normal form

$$
d s^{2}=|T|^{2}\left(d u^{2}+d v^{2}\right), \quad T \neq 0,
$$

by a mapping of class $C^{1}$, if there exists a factorization $d s^{2}=\omega \bar{\omega}$, where 
$\omega=P d x+Q d y$ satisfies the conditions of (IV). It will be supposed below that $E, F, G$ are continuous.

For example, if (74) is of the form $d s^{2}=d x^{2}+G d y^{2}$, then a sufficient condition for the possibility of reducing $d s^{2}$ to the form (75) is that $G(x, y)$ satisfy a uniform Lipschitz condition with respect to $x$. For, in this case, $d s^{2}=\omega \bar{\omega}$, where $\omega=d x+i G^{1 / 2} d y$.

If $d s^{2}$ is an orthogonal form $d s^{2}=E d x^{2}+G d y^{2}$, so that

$$
d s^{2}=\left|E^{1 / 2} d x+i G^{1 / 2} d y\right|^{2},
$$

it is sufficient that $E, G$ satisfy uniform Lipschitz conditions with respect to $y$, $x$, respectively. More generally, it is sufficient that there exists a positive, continuous function $\mu$ such that $\mu^{2} E, \mu^{2} G$ satisfy uniform Lipschitz conditions with respect to $y, x$, respectively (since $d s^{2}=\mu^{-2}\left|\mu E^{1 / 2} d x+i \mu G^{1 / 2} d y\right|^{2}$ ).

The general case (74) can be factored as $d s^{2}=\mu^{-2} \omega \bar{\omega}$, where

$$
\begin{aligned}
\mu^{-1} G^{1 / 2} \omega= & \{(F \cos \theta-W \sin \theta) d x+G \cos \theta d y\} \\
& +i\{(F \sin \theta+W \cos \theta) d x+G \sin \theta d y\},
\end{aligned}
$$

$W=\left(E G-F^{2}\right)^{1 / 2}$ and $\mu=\mu(x, y)>0, \theta=\theta(x, y)$ are arbitrary continuous functions. The choice $\mu \equiv 1, \theta \equiv 0$ gives

$$
\omega=\left\{\left(F / G^{1 / 2}\right) d x+G^{1 / 2} d y\right\}+i\left(E-\left(F / G^{1 / 2}\right)^{2}\right)^{1 / 2} d x,
$$

so that (74) can be reduced to the form (75) if, for example, $E_{y},\left(F / G^{1 / 2}\right)_{x}$, $\left(F / G^{1 / 2}\right)_{y}, G_{x}$ exist, and are bounded.

The remarks of this section answer in part questions raised by Wintner $[15]$.

\section{Appendix}

1. The object of this appendix is to prove the following assertion:

$\left(^{*}\right)$ On the circle $x^{2}+y^{2}<1$, let the coefficients of the quadratic differential form

$$
\alpha=L(x, y) d x^{2}+2 M(x, y) d x d y+N(x, y) d y^{2}
$$

be real-valued, of class $C^{1}$ and satisfy

$$
L N-M^{2}<0 .
$$

In addition, suppose that $\alpha$ has a continuous curvature $K(x, y)$. Then there exists a pair of functions

$$
x=x(u, v), \quad y=y(u, v)
$$

of class $C^{2}$ on a circle $u^{2}+v^{2}<1$, satisfying $x(0,0)=y(0,0)=0$, and transforming (1) into the normal form

$$
\alpha=2 T(u, v) d u d v, \quad T(u, v)>0 .
$$


This is the analogue of the assertion of [4] in the elliptic case, where (2) and (4) are replaced by $L N-M^{2}>0$ and $\alpha=T(u, v)\left(d u^{2}+d v^{2}\right)$, respectively. In this elliptic case, every mapping (3) bringing $\alpha$ into the conformal normal form is of class $C^{2}$; above, in the hyperbolic case, it is only stated that some mappings (3) achieving the normal form (4) are of class $C^{2}$ (and, in fact, there are some of class $C^{1}$ which are not of class $C^{2}$ ).

If $n=1$ in the assumption $\alpha \in C^{n}, K \in C^{n-1}$ and the assertion $x, y \in C^{n+1}$ of $\left({ }^{*}\right)$ is replaced by an $n>1$, then $\left(^{*}\right)$ becomes a theorem of Wintner [5, pp. 853-854]. Assertion $\left(^{*}\right)$ (with $n=1$ ) is conjectured to be true in [5]. As pointed out in [5], corollaries of $\left(^{*}\right)$ are the following theorems:

$\left(^{* *}\right.$ ) If $S$ is a (small piece of a) surface of class $C^{3}$ of negative Gaussian curvature, then $S$ has a parametrization of class $C^{2}$ in which the parametric lines are asymptotic curves.

$(* * *)$ If $S$ is a (small piece of a) surface of class $C^{3}$ without umbilical points, then $S$ has a parametrization of class $C^{2}$ in which the parametric lines are lines of curvature.

For analogues when $S \in C^{n}, n>3$, cf. [5]. If $S \in C^{2}$ (instead of $C^{3}$ ) and has a negative curvature, then $S$ need not possess asymptotic line parametrizations; see [8, p. 150], and for a sharper result, [2, p. 490]. On the other hand, $\left({ }^{*}\right)$ is final in the sense that if an $S$ possesses a $C^{3}$ asymptotic parametrization, then $S$ has $C^{4}$ (nonasymptotic) parametrizations; [1, p. 139].

2. When (2) holds and $L, M, N$ are of class $C^{1}$, then $\alpha$ has a factorization

$$
\alpha=2 \omega_{1} \omega_{2},
$$

where $\omega_{1}=P_{1} d x+Q_{1} d y, \omega_{2}=P_{2} d x+Q_{2} d y$ are linear differential forms with realvalued coefficients of class $C^{1}$; cf. $\$ 6$ above. The exterior product $\omega_{1 \wedge} \omega_{2}$ is defined to be $\left(P_{1} Q_{2}-P_{2} Q_{1}\right) d x d y$. The assumption (2) implies that $\omega_{1}, \omega_{2}$ are linearly independent, that is, that $P_{1} Q_{2}=P_{2} Q_{1} \neq 0$.

If $\omega_{1}, \omega_{2}$ are real, linearly independent forms with exterior derivatives and continuous densities (as is the case above when $P_{1}, Q_{1}, P_{2}, Q_{2} \in C^{1}$ ), then there exists a unique, real, linear differential form $\omega_{12}=P_{12} d x+Q_{12} d y$ with continuous coefficients satisfying

$$
d \omega_{1}=\omega_{12} \wedge \omega_{1}, \quad d \omega_{2}=\omega_{2} \wedge \omega_{12} .
$$

Suppose that the form $\omega_{12}$ has an exterior derivative with a continuous density $\delta\left(\omega_{12}\right)$ relative to $d x d y$, then (1) or (5) is said to have a continuous curvature $K(x, y)$ defined by

$$
K=\delta\left(\omega_{12}\right) /\left(P_{1} Q_{2}-P_{2} Q_{1}\right)
$$

or

$$
d \omega_{12}=K \omega_{1} \wedge \omega_{2}
$$

This is equivalent to the standard definition of curvature of (1) when $L, M, N$ 
are of class $C^{2}$ (in which case, $\omega_{12}$ can be chosen of class $C^{1}$ and $\delta\left(\omega_{12}\right)=Q_{12 x}$ $\left.-P_{12 y}\right)$.

When (1) is the second fundamental form of a surface $S$ belonging to a parametrization of class $C^{3}$, then $L, M, N$ are of class $C^{1}$ and (1) possesses a continuous curvature. This is clear from the considerations of $[4, \mathrm{pp} .688-$ 689]. Correspondingly, on a surface $S$ of class $C^{3}$ without umbilical points, a quadratic differential form (1) determining the lines of curvature by the equation $\alpha=0$ can be chosen so that $L, M, N$ are of class $C^{1}$ and $\alpha$ possesses a continuous curvature. This can be verified by following the considerations of Weingarten (cf., e.g., [3, pp. 486-488]) without using a particular coordinate system; cf. also [5, pp. 861-862].

3. Proof of $\left({ }^{*}\right)$. It is clear that there exist one-to-one mappings (3) of class $C^{1}$ on $|u|<\epsilon,|v|<\epsilon$, satisfying $x(0,0)=y(0,0)=0$ of nonvanishing Jacobian, transforming $\omega_{1}, \omega_{2}$ into the normal forms

$$
\omega_{1}=T_{1} d u, \quad \omega_{2}=T_{2} d v
$$$$
T_{1} T_{2}>0
$$

where the partial derivatives

$$
T_{1 v}, T_{2 u} \text { exist and are continuous; }
$$

cf., e.g., $\$ 5$ above. In view of (5), the relation (4) holds with

$$
T=T_{1} T_{2}>0 \text {. }
$$

It can be supposed that

$$
T(u, 0) \equiv T(0, v) \equiv 1 .
$$

For otherwise the variables $(u, v)$ can be replaced by new variables $\left(u^{\prime}, v^{\prime}\right)$ defined by

$$
u^{\prime}=T^{-1 / 2}(0,0) \int_{0}^{u} T(u, 0) d u, \quad v^{\prime}=T^{-1 / 2}(0,0) \int_{0}^{v} T(0, v) d v .
$$

The transformation $(u, v) \rightarrow\left(u^{\prime}, v^{\prime}\right)$ is of class $C^{1}$ and of nonvanishing Jacobian. Under this mapping, $\omega_{1}$ and $\omega_{2}$ become $\left(T_{1}(u, v) T^{1 / 2}(0,0) / T(u, 0)\right) d u^{\prime}$ and $\left(T_{2}(u, v) T^{1 / 2}(0,0) / T(0, v)\right) d v^{\prime}$. The product of the coefficients of $d u^{\prime}$ and $d v^{\prime}$ is, by (11), $T(u, v) T(0,0) / T(u, 0) T(0, v)$. This product is identically 1 if either $u=0$ or $v=0$, that is, if either $u^{\prime}=0$ or $v^{\prime}=0$. Thus, if $\left(u^{\prime}, v^{\prime}\right)$ are renamed to $(u, v)$, then (12) holds.

It will be shown that if the mapping (3) satisfies the normalization (12), then it is of class $C^{2}$.

Let $h=h(u, v)$ be the positive, continuous function satisfying

$$
T_{1}=h T^{1 / 2} \text { and } T_{2}=T^{1 / 2} / h \text {, i.e., } h=\left(T_{1} / T_{2}\right)^{1 / 2}>0 .
$$

Thus

$$
\omega_{1}=h T^{1 / 2} d u, \quad \omega_{2}=\left(T^{1 / 2} / h\right) d v .
$$


Then $d \omega_{1}=-\left(h T^{1 / 2}\right)_{v} d u d v=-\left(\left(h T^{1 / 2}\right)_{v} / T\right)\left(\omega_{1} \wedge \omega_{2}\right) ; \quad$ similarly, $\quad d \omega_{2}$ $=\left(\left(T^{1 / 2} / h\right)_{u} / T\right)\left(\omega_{1 \wedge} \omega_{2}\right)$. It follows from (6) that the unique form $\omega_{12}$ is given by $\omega_{12}=T^{-1}\left(\left(h T^{1 / 2}\right)_{v} \omega_{2}-\left(T^{1 / 2} / h\right)_{u} \omega_{1}\right)$; hence, from (14), by

$$
\omega_{12}=-\left(\log \left(T^{1 / 2} / h\right)\right)_{u} d u+\left(\log \left(T^{1 / 2} h\right)\right)_{v} d v .
$$

The relation (8) means that

$$
\int_{J} \omega_{12}=\iint_{E} K T d u d v
$$

holds for every domain $E$ bounded by a piecewise smooth Jordan curve $J$ in $|u|<\epsilon,|v|<\epsilon$.

Let $E$ denote the rectangle with vertices $(0,0),(u, 0),(u, v),(0, v)$, where $|u|<\epsilon,|v|<\epsilon$. Then (15) shows that the line integral in (16) becomes

$$
-\log T(u, v)+\log T(0, v)-\log T(0,0)+\log T(u, 0) .
$$

The normalization (12) and (16) give

$$
-\log T(u, v)=\int_{0}^{v} \int_{0}^{u} K T d u d v .
$$

Since $K$ and $T$ are continuous functions of $(u, v)$, it follows that $T(u, v)$ is of class $C^{1}$. Thus, the mapping (3) of class $C^{1}$ transforms the indefinite form (1) with coefficients of class $C^{1}$ into the indefinite form (4) with coefficients of class $C^{1}$. The theorem (II) in [10, p. 348], implies that (3) is of class $C^{2}$. This proves $(*)$.

REMARK. Note that $T$ is not only of class $C^{1}$ but has a continuous, second mixed derivative $T_{u v}=T_{v u}$ and that, in $(u, v)$-coordinates, the curvature $K$ can be calculated from the standard equation $(\log T)_{u v}+K T=0$.

\section{REFERENCES}

1. L. Ahlfors, On quasi-conformal mappings, Journal d'Analyse Mathématique vol. 3 (1954) pp. 1-58, 207-208.

2. H. Cartan, Algebraic topology, Mimeographed notes, Harvard University, 1949.

3. E. Cartan, Leçons sur les invariants integraux, Paris, 1922.

4. S. S. Chern, P. Hartman and A. Wintner, On isothermic coordinates, Comment. Math. Helv. vol. 28 (1954) pp. 301-309.

5. G. Evans, An elliptic system corresponding to Poisson's equation, Acta Literarum ac Scientiarum (Szeged) vol. 6 (1932-1934) pp. 27-33. 730.

6. P. Hartman, On the local uniqueness of geodesics, Amer. J. Math. vol. 72 (1950) pp. $723-$

7. P. Hartman and A. Wintner, On the fundamental equations of differential geometry, ibid. vol. 72 (1950) pp. 757-774.

8. - On the asymptotic curves of a surface, ibid. vol. 73 (1951) pp. 149-172.

9. - On the local behavior of solutions of non-parabolic partial differential equations, ibid. vol. 76 (1954) pp. 449-476.

10. - Regular binary Pfaffians and non-parabolic partial differential equations, Rend. Circ. Mat. Palermo vol. 3 (1954) pp. 347-362. 
11. M. Lavrentieff, $A$ fundamental theorem of the theory of quasi-conformal mappings of 2dimensional regions, Bull. Acad. Sci. URSS. Ser. Math. vol. 12 (1948) pp. 513-514; Amer. Math. Soc. Translations, no. 29.

12. C. B. Morrey, On the solutions of quasi-linear elliptic partial differential equations, Trans. Amer. Math. Soc. vol. 43 (1938) pp. 126-166.

13. - Multiple integral problems in the calculus of variations and related topics, Berkeley, 1943.

14. L. Nirenberg, On nonlinear partial differential equations and Hölder continuity, Communications on Pure and Applied Mathematics vol. 6 (1953) pp. 103-156.

15. A. Wintner, Remarks on binary Riemannian metrics, Rend. Circ. Mat. Palermo vol. 5 (1956) pp. 59-72.

\section{APPENDIX}

1. P. Hartman and A. Wintner, On the curvatures of a surface, Amer. J. Math. vol. 75 (1953) pp. 127-141.

2. - On asymptotic parametrizations, ibid. vol. 75 (1953) pp. 488-496.

3. V. Hlavaty, Differentialgeometrie, Groningen, 1939.

4. A. Wintner, On the local role of the theory of the logarithmic potential in differential geometry, Amer. J. Math. vol. 75 (1953) pp. 679-690.

5. - On indefinite binary Riemannian metrics, ibid. vol. 77 (1955) pp. 853-867.

The Johns Hopkins University,

BALTIMORE, MARYLAND 